\title{
Wireless Real-time IP Services Enabled by Header Compression
}

\author{
Krister Svanbro ${ }^{\dagger}$, Hans Hannu ${ }^{\dagger}$, Lars-Erik Jonsson ${ }^{\dagger}$, Mikael Degermark ${ }^{\neq}$ \\ $t$ Ericsson Research \\ Ericsson Erisoft $\mathrm{AB}$ \\ Box 920, SE-971 28 Luleå, Sweden \\ $\neq$ Dept. of CS \& EE \\ Luleå University of Technology \\ SE-971 87 Luleå, Sweden
}

\begin{abstract}
The world of telecommunications is currently going through a shift of paradigm from circuit switched, connection oriented information transfer towards packet switched, connection-less transfer. For application independence and to decrease costs for transport and switching it is attractive to go IP all the way over the air interface to the end user equipment, i.e., to not terminate the IP protocols before the air interface. A major reason to avoid using voice over IP over the air interface has, up to now, been the relatively large overhead imposed by the IP/UDP/RTP headers of voice packets. This paper presents a novel header compression scheme, ROCCO, that can compress the large headers to a minimum of one octet and is robust against the errors imposed by cellular links. Its performance is excellent both in terms of robustness and compression efficiency. Moreover, system capacity simulations are presented, which show that ROCCO gives significantly higher system capacity than the other current header compression candidate, CRTP. Finally it is argued that with ROCCO voice over IP over wireless becomes feasible from a spectrum-efficiency point of view.
\end{abstract}

\section{Introduction}

The telecom industry is currently starting to embrace the IP protocol suite as the choice of technology for the future. For application independence and to decrease costs for transport and switching it is attractive to go IP all the way over the air interface to the end user equipment, i.e., to not terminate the IP protocols before the air interface [1]. IP all the way enables IP service flexibility, that is, there are no dependencies between applications and the wireless access network and a base is created where many players can participate and develop new applications. This should be compared with present-day cellular services, which are vertically integrated and optimized - resulting in very high radio performance but low service flexibility. Cellular access networks have been optimized for quality and spectrum efficiency resulting in a highly efficient circuit switched voice service. The requirements in terms of spectrum efficiency and voice quality for the voice over IP service in future all IP cellular systems will reasonable be similar to current requirements on the circuit switched service. This introduces new challenges when realizing voice over IP over wireless. One fundamental challenge is to reduce the IP header related overhead over the relatively error prone and narrow band cellular channels, while maintaining the transparency of all header fields.
A major problem with voice over IP over wireless is the large headers of the protocols used when sending speech data over the Internet. An IPv4 packet with speech data will have an IP header, a UDP header, and an RTP header making a total of $20+8+12=40$ octets. With IPv6, the IP header is 40 octets for a total of 60 octets. The size of the speech data depends on the codec, it can be 15-30 octets. These numbers present a major reason for terminating the IP protocols before the air interface: the IP/UDP/RTP headers require a higher bit rate and would cause inefficient use of the expensive radio spectrum. However, it is possible to overcome this problem with a technique called header compression [2][5][6].

This paper presents a novel header compression scheme, ROCCO, which is suitable for cellular usage. The ROCCO scheme is presented together with link and system performance figures. In chapter II. the general techniques and properties of header compression is presented. Chapter III. gives the principles and functionality of the ROCCO scheme. Chapter IV. is a brief overview of how header compression schemes can be realized in $3^{\text {rd }}$ generation cellular systems. Chapter V. and VI. show header compression performance on the link and system level respectively, while conclusions are drawn in chapter VII.

\section{Header Compression}

While all header information in a voice packet is needed, there is a high degree of redundancy between header fields in the headers of consecutive packets belonging to the same packet stream. This observation is the basis for header compression algorithms. These algorithms maintain a context - essentially the uncompressed version of the last header transmitted - at both ends of the channel over which header compression is performed. Compressed headers carry changes to the context; static header fields need not be transmitted at all, and fields where the change is small are updated using few bits. When frames are lost or damaged over the channel, as they can be for cellular links, the context on the downstream side may not be updated properly and decompression of subsequent compressed headers will produce incorrect headers. Thus, header compression schemes must have mechanisms for installing context, for detecting when the context is out of date, and for repairing the downstream context when it is out of date. 
Within the IETF (Internet Engineering Task Force) several header compression algorithms are being standardized. For real-time IP services, CRTP [5] is relevant, and can compress 40 octet IPv4/UDP/RTP headers to a minimum of 2 octets. For context repair, CRTP relies on there being an upstream link over which the decompressor sends requests for updating headers. While the context is out of date, all packets received by the decompressor will be lost since the headers cannot be decompressed. The round trip time over the link will thus limit the efficiency of the context repair mechanism. CRTP performance over cellular links has been evaluated in [7], which found that the compression ratio of CRTP is sufficient, but the packet loss rate for CRTP over realistic cellular links is too high. A viable header compression scheme for voice over IP over wireless must be less fragile than CRTP but cannot be less efficient. Chapter V. elaborates further on CRTP performance over cellular links.

There are three basic requirements on a header compression scheme that is to be used over a cellular link:

1. The header compression scheme must be robust against the loss pattern of the cellular link, i.e. it must be able to tolerate loss between the compressor and decompressor.

2. The header compression scheme must have a high compression ratio. The compressed headers must be small to enable efficient usage of the scarce spectrum.

3. The header compression scheme must be loss-less, i.e. a header must be decompressed to be identical to the header before compression. The probability for producing erroneous headers must be kept below a very small threshold.

\section{RObust Checksum-based header COmpression (ROCCO)}

A header compression scheme with a high degree of compression and robustness suitable for cellular usage has been developed and proposed for standardization in the IETF by the authors. The scheme is called ROCCO (RObust Checksum-based header COmpression) [2] and is heavily geared towards local context repair.

A checksum covering the original (uncompressed) header is included in the compressed header to introduce a reliable way to detect when the context is out of date, and when an attempt to repair the context locally has succeeded. Figure 1 illustrates the basic principle of ROCCO. The checksum covering the original full header is sent to the decompressor in the compressed header. This enables local repair of context by means of repeated header reconstruction attempts and verifications. It also provides continuos verification of the context of the decompressor. Hence, the probability for producing erroneous decompressed headers is very small when using ROCCO.
To be able to achieve sufficiently good compression and robustness performance without losing generality, a concept of compression profiles have been introduced with ROCCO. Different compression profiles handle different kinds of RTP-streams and channel conditions to achieve as high performance as possible. Profiles are being developed for voice and video streams, taking cellular channel properties into account. However, a general all-purpose profile could of course also be developed.

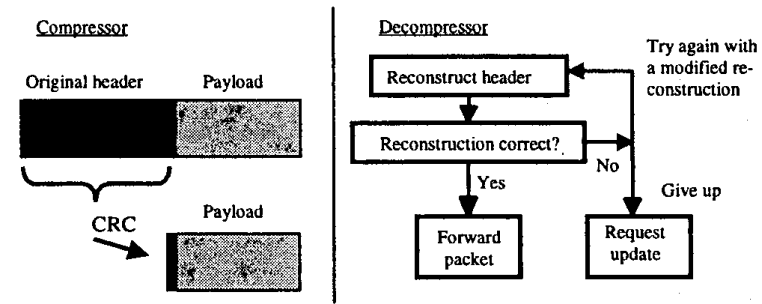

Figure 1. Schematic view of ROCCO header compression and decompression

In addition to the ROCCO header checksum, a code included in the compressed header provides the decompressor with hints about how the header fields have changed, e.g., due to loss over the cellular link. For the voice over IP profiles of ROCCO this code contains enough information to enable local repair of the context even after several (up to 26) consecutive lost packets. The ROCCO profile for voice over IP with maximum compression ratio compresses the RTP/UDP/IP headers to a minimum size of 1 octet. The average header size will, however, be slightly larger depending on channel conditions and header field change behavior.

The ability of ROCCO to locally repair the context virtually eliminates the negative effect of long round trip times on header compression robustness. ROCCO also has a high compression ratio that enables spectrum efficient voice over IP. Furthermore, the continuous verification of the decompressor context will in practice eliminate the possibility of producing erroneous decompressed headers.

\section{Realization of Header Compression in Third Generation Cellular Systems}

The global Third Generation Partnership Project (3GPP) [3] produces Technical Specifications for a $3^{\text {rd }}$ Generation (3G) Cellular System based on the evolved GSM core networks and the supported radio access technologies (i.e. UTRA both FDD and TDD modes). In the first phase of 3GPP standards there will be support for header compression of UDP/IP and TCP/IP headers, e.g. RFC2507. For future releases it is likely that there will also be support for robust and efficient header compression for real-time services such as voice, which entails compressing the RTP/UDP/IP header. This will ensure efficient 
realization of voice over IP all the way over the air interface to the user equipment.

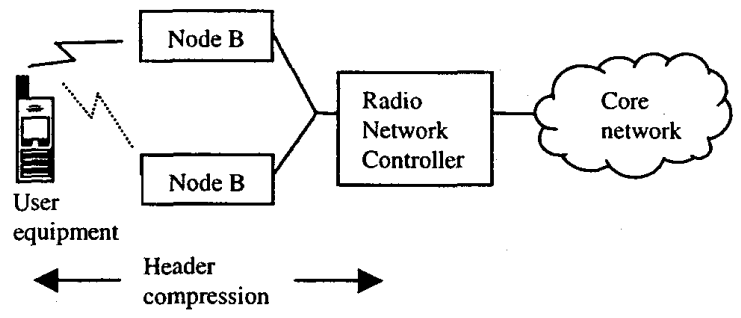

Figure 2. View of a $3 G$ system and between which two points header compression may be conducted

In a 3G system it is likely that the header compression endpoints will reside in the mobile terminal and in the radio network controller when compressing headers over the air interface, see figure 2. For a WCDMA system it is beneficial to have the compression end-point in the radio network controller since it is in the radio network controller combining for soft hand-over and ARQ is made. Further, it is beneficial if the header compression endpoints are close, to avoid long control loop delays. When all these things are considered, it is realistic to assume that the header compression end-points will be in the terminal and the radio network controller.

Traditionally, header compression schemes have relied on some functionality in the link layer carrying the compressed headers. It has been assumed that the link layer can provide the header compression scheme with error detection, framing, packet length indication and packet type identification. This functionality is of course also accessible in a $3 \mathrm{G}$ cellular system: error detection can be provided with layer 1 (physical layer) checksums while framing and packet length notification are inherent functions of layer 2 (MAC/RLC). With ROCCO, the packet type identification is incorporated in the header compression scheme and, thus this functionality is not needed from the link layer. This reduces the necessary per packet overhead with approximately one byte compared to a scheme without packet type identification, e.g. CRTP. Further, there should also be functionality in the link layer for negotiating header compression parameters in callsetup phases.

One property of a $3 \mathrm{G}$ system which is of importance for header compression schemes is the concept of logically separated radio bearers in contradiction to completely shared channels such as for example Ethernet. In for example [5] and [6], context identifiers (CIDs) are used to identify which context that should be used to decompress a compressed header. In a $3 G$ cellular system there has already been a de-multiplexing of the traffic onto different radio bearers, and this separation drastically reduces the need for context identification.

\section{Header Compression Performance on Wireless Links}

Detailed link level simulations have been made for ROCCO and CRTP over both WCDMA and EDGE channels. The simulated scenario is shown in Figure 3. A source generates speech RTP packets, which are sent over a network including various wired and wireless links. The last link on the path to the end system is a cellular link, over which packets with compressed IP/UDP/RTP headers are sent from the compressor (HC) to the decompressor (HD). Two profiles are evaluated for ROCCO, one profile with a minimum header size of 2 octets and another improved profile with a minimum header size of 1 octet. The former manages multiple contexts on a shared link while the latter is optimized for compression efficiency.

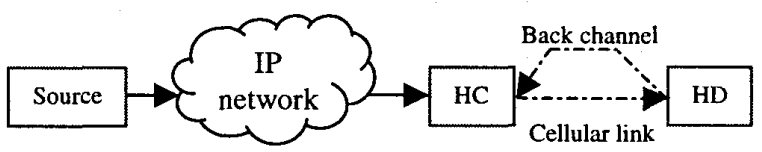

Figure 3. Scenario for link level simulations

The source in the simulations models an AMR speech codec running in $12.2 \mathrm{kbit} / \mathrm{s}$ mode with silence suppression. The speech and silence periods are both exponentially distributed with a mean value of one second. The packet stream suffers from a $0.5 \%$ uniformly distributed packet loss before the compression point, i.e. in a prior IP network. There is no reordering of packets. CRTP needs to have the packet type identification provided by the link layer whereas ROCCO has the packet type identification integrated. Hence, one octet of link layer overhead is added the for CRTP case. This octet is not included in the presented results.

\begin{tabular}{|c|c|}
\hline WCDMA link & EDGE link \\
\hline Ideal fast power control & Ideal frequency hopping \\
\hline $\begin{array}{c}\text { Gaussian white noise } \\
\text { interference }\end{array}$ & Single interferer \\
\hline $1 / 3$ convolutional coding & Code rate: $0.2(250 / 1264)$ \\
\hline $\begin{array}{c}\text { Indoor to Outdoor pedestrian } \\
\text { A fading model }\end{array}$ & Typical Urban fading model \\
\hline $3 \mathrm{~km} / \mathrm{h}$ mobile speed & $3 \mathrm{~km} / \mathrm{h}$ mobile speed \\
\hline Spreading factor 128 & 8-PSK modulation \\
\hline
\end{tabular}

Table 1. Properties of the simulated links

Two cellular links are used in the simulations presented in this paper; an EDGE link and a WCDMA link. See table 1 for properties of the two links. The reported bit error rate, BER, is the BER seen by the link layer. That is, the BER visible to the header compression scheme. The back channel never damages the context request messages and the round trip time for the cellular link and the back 
channel is set to $120 \mathrm{~ms}$ (the time for 6 speech frames). The error free back channel favors CRTP over ROCCO, since CRTP relies on the back channel for context repair. To improve the context repair of CRTP multiple context requests and updates are sent in a row at a context error.

A packet is considered lost if it is not passed up to the application (speech codec). This can be due to three different reasons in this study:

1. A bit error has occurred in the (compressed) header.

2. A bit error has occurred in the link layer packet type identification (for the CRTP case only) or in the link layer checksum.

3. The header compression scheme has a faulty context and cannot decompress any received compressed header (context damage). Note: this can happen even if the compressed header is error free.

Hence, a packet with errors in the payload is not regarded as lost as long as it is deemed ok by the header compression scheme.

Figure 4 shows the packet loss distribution for the two header compression schemes over a WCDMA link. It is evident from this figure that the majority of the loss events with CRTP comes from its context repair mechanism, which is heavily dependent on the round trip time. ROCCO does not suffer from context damage as it can handle consecutive packet losses between the compressor and decompressor without producing a faulty context.

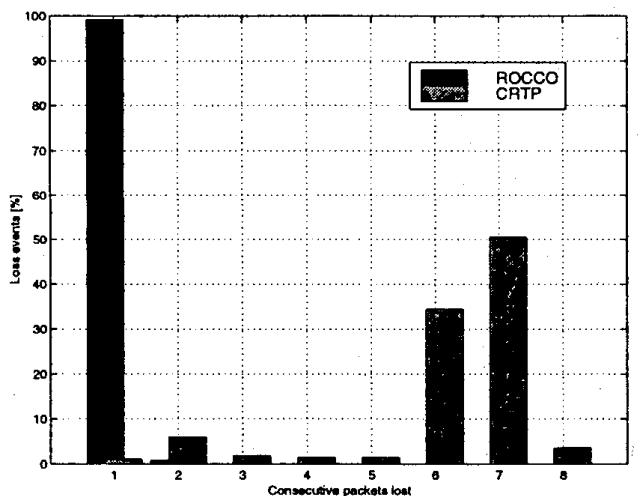

Figure 4. Distribution of packet loss for CRTP and ROCCO over a WCDMA link

Figure 5 shows the packet loss rate versus channel BER. The difference in robustness to high bit error rates is clearly visible for the two header compression schemes. A packet loss between a CRTP compressor and decompressor triggers a burst of additional packet losses due to CRTPs round trip based error recovery. This results in unacceptable packet loss rates for CRTP when the BER of the link approaches typical cellular BER. The robustness of ROCCO ensures that a valid context is maintained even after several consecutive lost packet. This virtually eliminates packet losses due to context damage. The packet loss rate of CRTP is about 6 times higher than for ROCCO for a BER of $10^{-3}$ on the simulated cellular links.

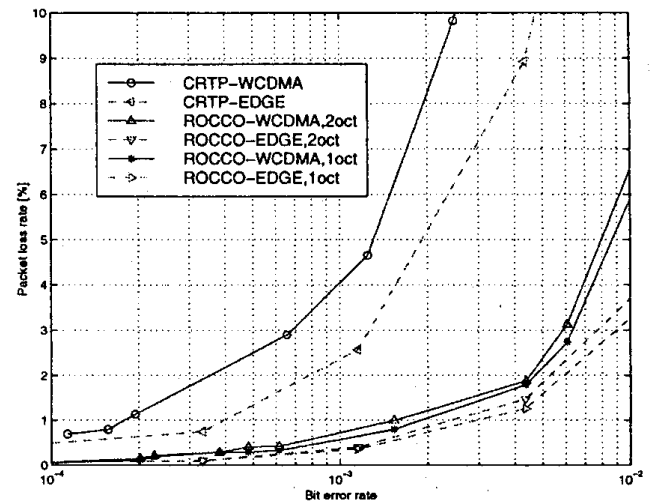

Figure 5. Packet loss rate versus channel BER

Figure 6 shows the average header size plotted against channel BER. The difference between CRTP and ROCCO is mainly that the latter tolerates losing several consecutive packets before it needs a context update packet, while CRTP needs a context update for each loss event. Since the context updating headers are larger ( 17 bytes for CRTP) the average compressed header size for CRTP increases as the BER increases. The context is rarely damaged for ROCCO and thus, the average header size is just slightly larger than the minimum header size. Furthermore, ROCCO has a minimum header size of 1 octet while the minimum header size of CRTP is 2 octets. Hence, ROCCO introduces significantly less header overhead than CRTP in a cellular environment. For a bit error rate of $10^{-3}$, the average header size of CRTP is almost two times the average header size of ROCCO (when optimized for compression ratio).

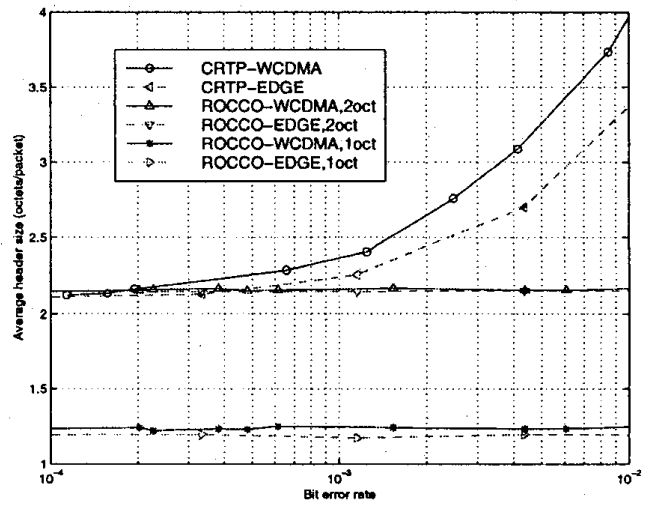

Figure 6. Average header size versus channel BER 
Simulations identical to the ones presented above have also been conducted for other types of channel models (i.e. WCDMA Vehicular A $50 \mathrm{~km} / \mathrm{h}$, Typical Urban $3 \mathrm{~km} / \mathrm{h}$ and EDGE Typical Urban without frequency hopping). These simulations show insignificant differences in link performance when average header size and packet loss rate is evaluated versus channel bit error rate. Hence, the variations of the BER pattern for the above-mentioned channels do not significantly affect header compression performance. Thus, it is feasible to evaluate header compression performance versus BER for different channel types and in a second step evaluate the effect on different BER requirements on carrier to interference ratio $(\mathrm{C} / \mathrm{I})$.

The relationship between bit error rate and carrier to interference ratio is, however, different for different channel types. Achieving a BER of $10^{-4}$ instead of $10^{-3}$ for the two simulated channels described in table 1 requires a raised $C / I$ requirement of approximately 1 and $3 \mathrm{~dB}$ for the WCDMA and the EDGE channel respectively. In figure 5 it can be seen that the packet loss rates for the EDGE channel are slightly lower than for the WCDMA channel for both CRTP and ROCCO. This is due to the burstier bit error characteristic of EDGE compared to the WCDMA channel with fast power control. However, since a raised requirement in BER for the EDGE channel results in a larger raise in $\mathrm{C} / \mathrm{I}$ requirement compared to WCDMA, it can be argued that a header compression scheme for EDGE has a higher requirement in terms of robustness to achieve sufficient system performance.

\section{Header Compression Impact on Cellular System Performance}

Using ROCCO instead of CRTP will increase the tolerance for channels with bit errors, and will result in an increased radio network capacity for a voice over IP over wireless system. Initial multi-cell simulations of a WCDMA system with voice over IP users only have been conducted. The simulations comprised several three-sector sites covering macro cells with a uniform geographical traffic distribution. The simulations include slow log-normal shadow fading, distance dependent path loss attenuation and ideal fast power control. The channel model used is according to the VehicularA [4] channel model for mobiles moving at $50 \mathrm{~km} / \mathrm{h}$. Equal error protection and un-equal error detection were applied to all studied cases. For the voice over IP packets the un-equal error detection covered only the (compressed) headers.

The simulations show the fundamental need of a header compression scheme when going IP all the way for voice over IP. If no header compression is applied and full headers are sent with every voice packet, a capacity decrease larger than $50 \%$ can be observed compared to the case where no header over-head is present. Hence, it is not possible to achieve spectrum efficiency for voice over IP comparable to the circuit switched solution without header compression.

The simulations also indicate that the capacity of a voice over IP system with ROCCO is rather close to the capacity of a circuit switched speech service. With ROCCO, the capacity of the voice over IP system is reduced with only $5 \%$ compared to the corresponding circuit switched service. However, the voice over IP application level signaling should also be taken into account.

Although the figures above are for a specific example, it is clear that using a highly robust and efficient header compression scheme, such as ROCCO, makes voice over IP over wireless an interesting and viable alternative to the traditional circuit switched cellular speech service.

\section{Conclusions}

IP all the way, also over the air-interface in cellular systems, enables IP service flexibility. However, going IP all the way in cellular systems for real-time IP services, such as voice over IP, introduces new challenges. One of the main challenges for real-time IP over wireless is how to transparently compress the relatively large IP header over error prone cellular links. The existing header compression scheme for real-time IP, CRTP, is not robust enough for cellular usage. A new header compression scheme, ROCCO, has been developed with cellular system characteristics in mind. System and link level simulations show that ROCCO has superior performance in both robustness and compression ratio compared to CRTP. Comparisons with corresponding circuit switched voice services show that with ROCCO, voice over IP over wireless becomes an interesting and viable alternative to the traditional circuit switched cellular speech service.

\section{References}

[1] K. Svanbro, "Voice over IP over Wireless Performance and Principles", Submitted to WTC/ISS2000, Birmingham, May 2000

[2] L-E. Jonsson, M. Degermark, H. Hannu, K. Svanbro, "RObust Checksum-based header COmpression (ROCCO)", Internet Draft (work in progress), January 2000

[3] The 3GPP Home Page, www.3gpp.org

[4] "Procedure for Evaluation of Transmission Technologies for PLMTS", ITU-R TG8-1,81/TEMP/233-E, September 1995

[5] S. Casner, V. Jacobson, "Compressing IP/UDP/RTP Headers for Low-Speed Serial Links", RFC 2508, February 1999

[6] Mikael Degermark, Bjorn Nordgren, Stephen Pink, "IP Header Compression", RFC 2507, February 1999

[7] M. Degermark, H. Hannu, L-E Jonsson, K. Svanbro, "CRTP over cellular radio links", Internet Draft (work in progress), December 1999 